\title{
Evaluating the Development of a SPECT Protocol in a Canadian Epilepsy Unit
}

\author{
J.G. Burneo, W. Vezina, J. Romsa, B.J. Smith, R.S. McLachlan
}

\begin{abstract}
Background: Functional neuroimaging can address some challenges of seizure localization, and sometimes preclude the need for EEG recording using intracranial electrodes. Ictal Single Photon Emission Computed Tomography (SPECT) has developed into an important tool in the presurgical evaluation of patients with medically-intractable localization-related epilepsy. The purpose of the study was to determine whether the development of a programme using trained nurses to perform ictal injections enabled a more efficient delivery of radiopharmaceuticals and therefore a greater sensitivity and specificity of outcome. Methods: In our epilepsy unit, nursing staff inject ${ }^{99} \mathrm{~m}$ Tc-HMPAO at bedside, during or at seizure onset. Brain SPECT is performed later on a gamma camera. Results: Since the implementation of the new protocol (February 2005), 57 scans have been performed: 22 ictal and 35 interictal. Latency of ictal injection was found to be 5-40 seconds (mean $19.7 \mathrm{sec}$, standard deviation (SD) 10.4). Only 20\% of reconstituted radiopharmaceutical vials were not used. Contamination rate was nil. Sixty three percent of SPECT studies were concordant with standard presurgical evaluation. Conclusion: The latency of injections and the percentage of unused vials indicated an efficient and effective protocol compared to the literature. Our results show that ictal SPECT can be a safe, noninvasive procedure performed on a routine basis in the epilepsy unit when appropriately trained support staff are incorporated into a structured multidisciplinary programme.
\end{abstract}

RÉSUMÉ: Évaluation du développement d'un protocole de TEMP dans une unité d'épilepsie au Canada. Contexte : La neuroimagerie fonctionnelle peut permettre de relever certains défis que pose la localisation des crises convulsives et obvier à la nécessité de recourir à l'enregistrement ÉEG effectué au moyen d'électrodes intracrâniennes. La TEMP ictale est devenue un outil important dans l'évaluation préchirurgicale de patients ayant une épilepsie localisée, réfractaire au traitement médical. Le but de cette étude était de déterminer si le développement d'un programme d'injection ictale par des infirmières ayant reçu une formation pertinente permettrait d'optimiser l'administration des substances radiopharmaceutiques et donc d'améliorer la sensibilité et la spécificité des résultats. Méthodes : Le personnel infirmier de notre unité d'épilepsie injecte le 99mTc-HMPAO au chevet du malade pendant ou au début de la crise convulsive. La TEMP du cerveau est ensuite réalisée par une caméra à rayons gamma. Résultats : Depuis l'implantation du nouveau protocole en février 2005, cinquante-sept scintigraphies ont été effectuées, soit 22 scintigraphies ictales et 35 interictales. La latence de l'injection ictale était de 5 à 40 secondes (moyenne 19,7 secondes, écart type 10,4 secondes). Seulement $20 \%$ des flacons de substance radiopharmaceutique reconstituée n'ont pas été utilisés. Le taux de contamination a été nul. Les résultats de soixante-trois pour cent des études TEMP concordaient avec ceux de l'évaluation préchirurgicale standard. Conclusion : Le temps de latence des injections et le pourcentage de flacons inutilisés indiquent qu'il s'agit d'un protocole efficace, ayant un bon rendement, comparé à ce qui est rapporté dans la littérature. Nos résultats démontrent que la TEMP ictale peut être une intervention sûre, non effractive, pratiquée de routine dans l'unité d'épilepsie quand un personnel de soutien formé est incorporé à un programme multidisciplinaire structuré.

Can. J. Neurol. Sci. 2007; 34: 225-229

Studies on the epidemiology of intractable epilepsy in the Canadian population are not available. ${ }^{1}$ However, extrapolating from studies done in the United States (US), the incidence is of approximately $6 / 100,000$ patients per year, translating to about 17,000 new cases destined to meet criteria of intractability annually. ${ }^{2}$ This group accounts for over $50 \%$ of the total cost of epilepsy. ${ }^{3}$ For many of these patients, epilepsy surgery offers the only opportunity to become completely or nearly free of seizures. The role of surgery for intractable epilepsy is rapidly changing. No longer an intervention of last resort, surgical therapy is often an earlier option to be considered because it may achieve seizure-free outcomes in patients otherwise intractable. ${ }^{4}$

A standard presurgical evaluation of a patient with intractable epilepsy consists of a video-EEG study performed in an epilepsy unit, an MRI of the brain, and a neuropsychological evaluation. If the localization of the epileptogenic focus is unequivocal, surgery can be performed. However in many cases this is not the situation and patients require more investigation using

From the Epilepsy Programme (JGB, RSM), Department of Nuclear Medicine (WV, JR), University of Western Ontario, London, ON, Canada; Comprehensive Epilepsy Program (BJS), Henry Ford Health Systems, Detroit, MI, USA.

Received August 8, 2006. ACCEPTED IN FINAL FORM JANuARY 21, 2007. Reprint requests to: Jorge G. Burneo, Epilepsy Programme, University of Western Ontario, B10-118, 339 Windermere Rd., London, Ontario, N6A 5A5, Canada. 
intracranially placed electrodes for further monitoring with EEG. When invasive tests such as intracranial EEG recordings are required, an important question is where precisely to place the electrodes. ${ }^{5}$ Noninvasive functional imaging techniques can provide additional information to help address these challenges of localization. Furthermore, it is possible that functional imaging may in some cases preclude the need for intracranial electrodes.

Ictal Single Photon Emission Computed Tomography (SPECT) has developed into a critical tool in epilepsy presurgical evaluations in a number of centers. This is true especially for patients in whom few other solid clues are present on which to develop a reasonable hypothesis that can guide intracranial placement of electrodes. ${ }^{5,6} \mathrm{~A}$ number of technical difficulties provide challenges for the implementation of a successful SPECT program in an epilepsy unit. This manuscript evaluates the development of such a program in the Epilepsy Unit at the London Health Sciences Centre.

\section{Methods}

\section{Patient sample}

All patients admitted to the Epilepsy Unit (EU) at London Health Sciences Centre for the purpose of prolonged video/EEG evaluation between February of 2005 and May 2006, were considered potential candidates for a SPECT study.

\section{Indication for SPECT}

The presurgical evaluation included a MRI of the brain, neuropsychological analysis, and prolonged video/EEG monitoring. In certain cases an intracarotid amobarbital procedure was performed and clinical psychology consultation was obtained. The performance of an ictal and/or interictal SPECT was at the physician's discretion, during or prior to admission.

\section{The Epilepsy Unit and the personnel}

The EU at The London Health Sciences Center consists of eight beds that are located in a single large area, surrounding the nursing station, in an Intensive Care Unit-like arrangement. All registered nurses working in the EU were taught about the indications for SPECT, as well as the protocol for injection, including radiation safety techniques practiced by the hospital. Injections are contraindicated if a nurse or a patient in the EU is pregnant, confirmed with beta human chorionic gonadotropin testing in serum, or if they are currently breast feeding.

\section{Protocol for injection}

Once admitted to the EU, patients requiring ictal SPECT as part of their evaluation need to have at least two seizures prior to the day of injection, in order to characterize events. ${ }^{99 \mathrm{~m}} \mathrm{Tc}-$ HMPAO (hexamethyl-propyleneamineoxime) is ordered by the physician (epileptologist) for injection the following day.

Early on the morning of injection, the dose of the radiopharmaceutical is prepared in the Department of Nuclear Medicine. A heparin lock attached to a 2-way stopcock is inserted into the patient's arm to facilitate rapid administration of the radiopharmaceutical. Technecium-99m HMPAO remains active prior to injection for four hours. The active form is quickly removed from the plasma compartment once injected.

The individual doses are transported to the EU in a lockable shielded syringe carrier, and upon arrival the heparin lock is tested for patency. One of the two nurses in the EU remains at patient's bedside and injects the HMPAO immediately at the onset of an aura or seizure. In some cases, in which the clinical seizure is not clear, the EEG onset is monitored from the nursing station by a physician or EEG technologist. Following the injection, the dose is flushed with saline. In order to calculate the latency of the ictal injection, the nurse doing the injection provides a verbal statement of when the radiopharmaceutical and the saline flush is completed.

If the patient does not have a seizure on the study day, the HMPAO is used for the interictal injection in order to decrease the amount of wasted material. The ictal injection is tried again the following day.

\section{SPECT SCANNING}

After injection is completed and the patient is stable, the Department of Nuclear medicine is contacted. Within the one hour following the injection the patient is taken for the scan. The patient is not disconnected from monitoring as the scans can be done with the electrodes still in place.

Brain SPECT is performed on a 2 headed gamma camera equipped with high resolution fan beam collimators. The data are acquired in a $128 \times 128$ matrix, with 64 stops at 25 seconds per stop. Images are reconstructed and can be displayed as slices in three orthogonal (axial, coronal, and sagittal) planes, with an additional temporal lobe plane, with images obtained obliquely along the long axis of the temporal lobes. Substraction analysis is available within the software (Delta program).

The examination of the images would reveal hypoperfusion ('cold spot') in the interictal state, and hyperperfusion ('hot spot') in the ictal state, in the location corresponding to the epileptogenic area. The interpretation is qualitative.

Finally, results from SPECT studies were compared with standard presurgical investigation (prolonged video-EEG, MRI of the brain, and neuropsychology evaluation). These results were classified as a) concordant, b) discordant, c) normal, if the studies were not useful in localizating a focus, and d) unknown, if the ictal attempt was unsuccessful. For patients with nonepileptic events, the ictal SPECT was defined to be useful, as long as the prolonged video-EEG did not reveal any electrographic changes during habitual spells.

\section{RESULTS}

Since the implementation of the new protocol in February of 2005 until May of 2006, 292 patients were admitted to the EU for prolonged video/EEG monitoring. Thirty five patients were identified as candidates for ictal SPECT.

There were 22 successful injections for ictal SPECT. A successful injection was defined as an injection done at or within seconds from the onset of the clinical seizure or event, and that allowed us to obtain a scan. Thirty-five interictal scans were performed. Thirty patients had injections for localization purposes; while three had injections to confirm diagnosis of nonepileptic events. The mean time to injection after clinical and/or 
EEG onset, as confirmed by EEG telemetry, was 19.7 seconds (Standard Deviation $=10.4$, range: $5-40)$. In those patients with seizures of brief duration, injections were within ten seconds of ictal onset. Three patients had continuous aura, the time was not taken in these cases.

There were 13 unsuccessful injections, related to the fact that the patients did not have seizures that day, during the working hours of the scanner (08:00 - 15:00). We did not experience any technical difficulties (e.g. subcutaneous extravasations of the radiopharmaceutical agent, blockage of the hep-lock).

Of the ictal studies done (determined by injection during ictal behavior or ictal pattern evident on EEG), $28 \%$ of patients had evidence of temporal lobe involvement, and $50 \%$ extratemporal involvement (hyperperfusion/hypoperfusion pattern to suggest ictal origin). Twenty four cases $(63 \%)$ had SPECT scans that were concordant with standard presurgical evaluation, one ictal SPECT was normal, and of the unsuccessful attempts, two cases revealed areas of hypoperfusion in interictal scans that were concordant with standard presurgical evaluation. The results were based on the visual impression of an area of hyperperfusion on the ictal scan and/or an area of hypoperfusion in the interictal study, by nuclear medicine physicians (WV, JR) and an epileptologist with training in this technique (JGB), and compared with clinical, MRI, electrophysiological data, subtraction analysis, and in some cases of co-registration to MRI (see Figures 1 and 2).

In this time-period, 95 vials were prepared, 19 (20\%) were returned to the Department of Nuclear Medicine unused. Our radioactive contamination rate was nil.

\section{Discussion}

The value of ictal SPECT in the presurgical evaluation of epilepsy cannot be underestimated. Single-photon-emission computed tomography is the only modality practically suited for imaging brain activity changes during an actual seizure.
Although there may be exceptions occasionally, in the clinical setting other functional imaging modalities such as PET and fMRI remain confined to detecting metabolic or blood flow derangements seen during the interictal rather than ictal state. The localizing information from successful studies is considerably more accurate than for interictal data alone, ictal SPECT scans can be extremely valuable in the assessment of all localization-related partial epilepsies.

Limitations that remain with ictal SPECT, center mainly on the logistics of successfully injecting the radiopharmaceutical as early as possible after onset of a seizure. The success of an ictal scan depends significantly on the injection latency. During a delay between seizure onset and injection of the radiopharmaceutical tracer, seizure activity may spread and result in false lateralization or localization on ictal SPECT. Injection times of less than 60 seconds from the onset of ictal discharge have been found to be sufficient to catch ictal hyperperfusion at the epileptogenic zones. ${ }^{7,8}$ Another consequence of the difficult logistics associated with ictal SPECT is the high cost of resources necessary to have health care staff sitting at the patient's bedside waiting for the onset of a seizure. Some centers, with the help of basic engineering groups, have devised and implemented automated or semiautomated injection systems. ${ }^{9}$ These operational issues remain an area that needs to be improved to reduce cost, improve accuracy, and ultimately bring this true advance in presurgical evaluation to more patients. Other concerns include limitation in flexibility in timing of injection, additional financial burden, the need for personnel to confirm the event and initiate the pump injection, as well as the need to train/educate staff regarding radiation handling and safety. It also increases the risk of contamination. ${ }^{10}$

It is clear that multidisciplinary cooperation is required for this type of endeavor, ${ }^{10}$ and that has been achieved in our center. Moreover, the layout of the unit has contributed to the successful

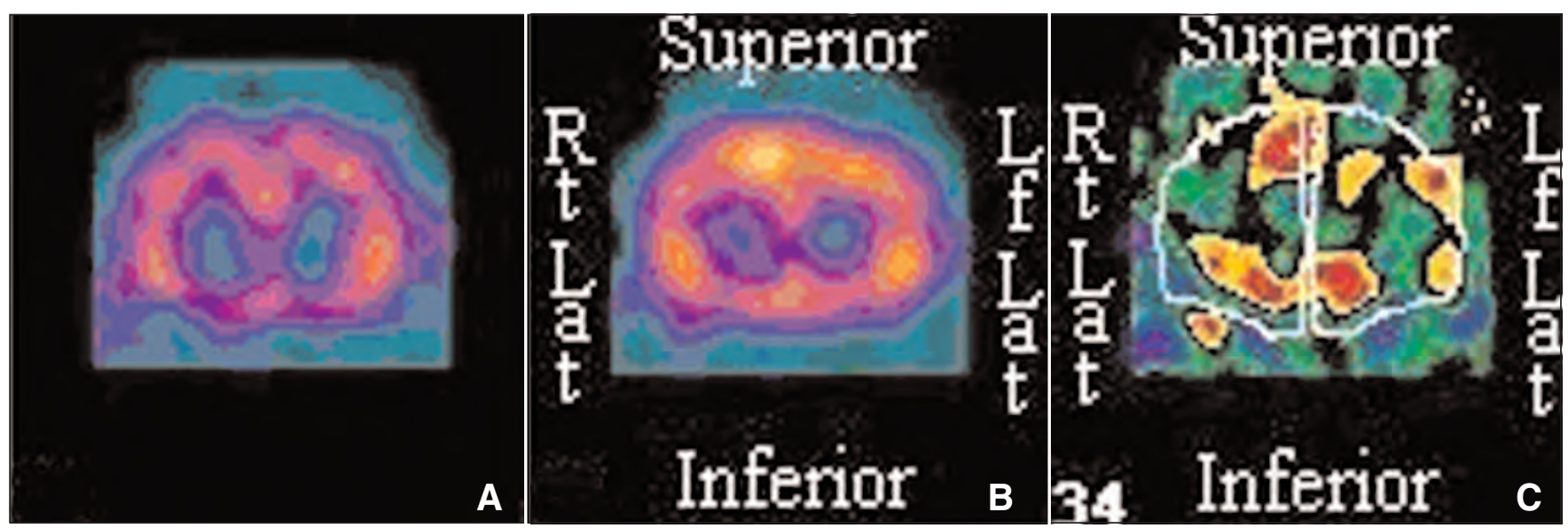

Figure 1: Interictal (left), ictal (center), and subtraction analysis (right) scans of a patient with left frontal lobe epilepsy. The interictal scan revealed normoperfusion of the frontal lobes, the ictal scan hyperperfusion over the left frontal region, the subtraction analysis (Subtraction $=$ Ictal interictal) confirmed the localization of the epileptogenic area. The injection in this patient was done within the initial 5 seconds following the seizure onset. 


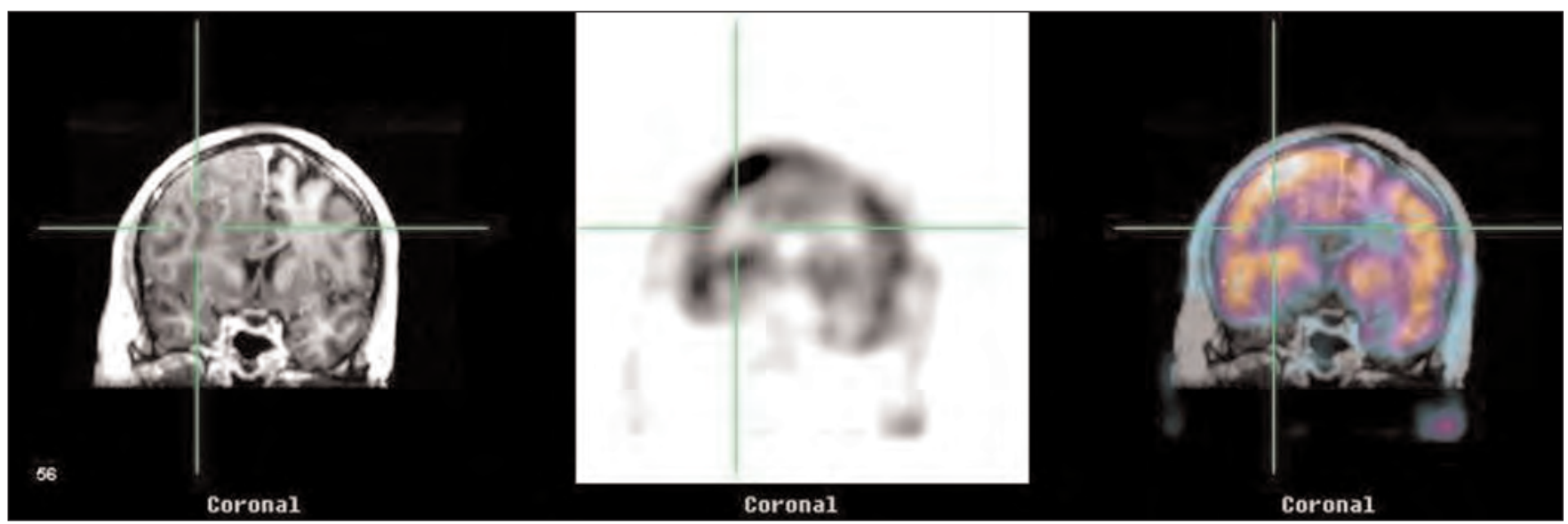

Figure 2:Coronal section of an MRI of the brain (far left picture) in a patient with a right frontal oligodendroglioma and history of focal seizures resistant to antiepileptic medications. Ictal SPECT (center) revealed an area of hyperfusion over the right frontal convexity outside the tumor area. Co-registration of SPECT findings onto MRI is shown on the far right.

latency times obtained since the implementation of the protocol. Our mean time for injection after the onset of the clinical seizure indicates a very efficient and effective protocol. In some centers, the personnel that perform the ictal injection are EEG technologists, ${ }^{10}$ dedicated research fellows, ${ }^{11}$ and even epileptologists. ${ }^{12}$ In our center, the advantage of having the nurse deliver the radiopharmaceutical resides in the fact that they are familiar with the patient's events and they have a greater experience in the management of intravenous administration of medications. This may be reflected in the absence of any contamination in our series.

Another contributor to the success in the implementation of the protocol is that the radiopharmaceutical does not need to be reconstituted prior to injection. Dose preparation is done in advance in the Department of Nuclear Medicine.

Unsuccessful injections due to the lack of events during the day planned for the delivery of the radiopharmaceutical still remain a problem. Our rate of unused reconstituted radiopharmaceutical material was low, but still 20 percent of the vials were returned. A limited amount of doses are requested, and that happens only after two seizures are witnessed in the EU. If the patient does not have a seizure, the dose is used for the interictal study. Another caveat is that we only order one dose per day; this is because we only have two nurses covering the unit.

Although the layout of the unit is advantageous for this protocol, it has some drawbacks. Injections cannot be done if another patient in a neighboring bed is pregnant. We found this to be rare, as we do not to admit pregnant patients to the EU.

In terms of cost, in the Province of Ontario, the performance of a SPECT scan includes a technical fee of approximately 45 CAD, a professional fee (nuclear medicine physician) of 27 $\mathrm{CAD}$, one vial of HMPAO is $340 \mathrm{CAD}$, and the Technetium needed for the stabilization of the HMPAO which approximately is 50 CAD. Making a total of approximately 460 CAD per scan, an amount of money that is substantially lower when compared with the costs of a PET scan. Having a nurse at bedside performing the injection, did not increase the costs, as they agreed to include this activity as part of their routine.

Finally, the results from the SPECT studies done in our center showed concordance with standard presurgical evaluation in $63 \%$ of cases; making the study a valuable one, and another tool in the armamentarium of presurgical non-invasive techniques for the localization of the epileptogenic area in patients with therapy resistant focal epilepsy.

In conclusion, this protocol has been effectively implemented and has allowed the performance of ictal SPECT studies with higher rates of success and lower rates of complications compared to what have been published in the literature.

\section{ACKNOWLEDGEMENTS}

The authors thank GE Healthcare Medical Diagnostics for supplying the HMPAO kits as a grant-in-kind, and the nurses at the epilepsy unit.

\section{REFERENCES}

1. Burneo JG, Tellez-Zenteno J, Wiebe S. Understanding the burden of epilepsy in Latin America: a systematic review of its prevalence and incidence. Epilep Res. 2005;66(1-3):63-74.

2. Hauser WA, Hesdorffer DC. Epidemiology of intractable epilepsy. In: Luders HO, Comair YG, editors. Epilepsy Surgery. 2nd ed. Philadelphia: Lippincott Williams \& Wilkins; 2001: p. 55-61.

3. Begley CE, Annegers JF, Lairson DR, Reynolds TF, Hauser WA. Cost of epilepsy in the United States: a model based on incidence and prognosis. Epilepsia. 1994;35(6):1230-43.

4. Wiebe S. Early epilepsy surgery. Curr Neurol Neurosci Rep. 2004:4(4):315-20.

5. Knowlton RC, Lawn ND, Mountz JM, Kuzniecky RI. Ictal SPECT analysis in epilepsy: subtraction and statistical parametric mapping techniques. Neurology. 2004;63(1):10-5.

6. Kuzniecky R, Mountz JM, Thomas F. Ictal 99mTc HM-PAO brain single-photon emission computed tomography in electroencephalographic nonlocalizable partial seizures. J 
Neuroimaging. 1993;3(2):100-2.

7. Newton MR, Berkovic SF, Austin MC, Rowe CC, McKay WJ, Bladin PF. Postictal switch in blood flow distribution and temporal lobe seizures. J Neurol Neurosurg Psychiatry. 1992;55(10):891-4.

8. Rowe CC, Berkovic SF, Austin MC, McKay WJ, Bladin PF. Patterns of postictal cerebral blood flow in temporal lobe epilepsy: qualitative and quantitative analysis. Neurology. 1991;41(7):1096-103.

9. Vanbilloen H, Dupont P, Mesotten L, et al. Simple design for rapid self-injection ictal SPET during aura. Eur J Nucl Med. 1999;26(10):1380-1.
10. Smith BJ, Karvelis KC, Cronan S, Porter W, Smith L, Pantelic MV, et al. Developing an effective program to complete ictal SPECT in the epilepsy monitoring unit. Epilepsy Res. 1999;33(2-3): 189-97.

11. Newton MR, Berkovic SF, Austin MC, Rowe CC, McKay WJ, Bladin PF. Ictal postictal and interictal single-photon emission tomography in the lateralization of temporal lobe epilepsy. Eur J Nucl Med. 1994;21(10):1067-71.

12. Rowe CC, Berkovic SF, Sia ST, Austin M, McKay WJ, Kalnins $\mathrm{RM}$, et al. Localization of epileptic foci with postictal single photon emission computed tomography. Ann Neurol. 1989;26(5):660-8. 